\title{
Neurogenic heterotopic ossification after a stroke: diagnostic and radiological challenges
}

\author{
Chong Han $\underline{\text { Pek }}{ }^{1}$, MBBS, MMed, Mei Chin $\underline{\text { Lim }}^{2}$, MBBS, FRCR, Ren $\underline{\text { Yong }}{ }^{1}$, MBBS, MMed, Ho Poh Wong ${ }^{1}$, MBBS, FAMS
}

\begin{abstract}
Heterotopic ossification (HO) is the aberrant formation of ectopic bone within the soft tissues, of which the aetiology is usually either traumatic or neurogenic. Neurogenic $\mathrm{HO}$ is a known but uncommon complication that occurs after a cerebral or spinal insult. The condition may present with a spectrum of symptoms and is often difficult to diagnose clinically. Although different imaging modalities have been used to diagnose HO, clinicians and radiologists may occasionally encounter radiological features of HO that may mimic other disease conditions. We herein report a rare case of neurogenic $\mathrm{HO}$ occurring in the non-paretic limb of a patient, and the diagnostic and radiological challenges encountered.
\end{abstract}

Keywords: ankylosis, heterotopic ossification, hip, neurogenic

\section{INTRODUCTION}

Heterotopic ossification $(\mathrm{HO})$ is defined as the aberrant formation of ectopic bone containing bone marrow within the soft tissues. ${ }^{(1,2)}$ Various forms of $\mathrm{HO}$ have been described - mainly traumatic and neurogenic in aetiology. Neurogenic $\mathrm{HO}$ is usually seen after insults such as spinal cord injury, traumatic brain injury, stroke and cerebral anoxia. ${ }^{(1)}$

Despite its known association with traumatic and neurogenic aetiology, the pathogenesis of $\mathrm{HO}$ remains poorly understood. In the case of neurogenic $\mathrm{HO}$, it has been hypothesised that prolonged immobilisation and subsequent manipulation of joints with force to preserve the range of motion (ROM) are the key causative factors. ${ }^{(3-6)}$ Other reports have hypothesised that damage to the central nervous system (CNS) results in abnormal activation of factors such as bone morphogenic protein, or systemic factors such as prostaglandin $\mathrm{E}_{2} \cdot{ }^{(1)}$ These factors could possibly stimulate mesenchymal cells to mature into osteoblasts, resulting in the formation of bone in tissues around the joint.

The occurrence of $\mathrm{HO}$ in post-stroke hemiplegic patients is rare. In Crawford et al's study, the incidence of $\mathrm{HO}$ after stroke was reported to be $0.5 \%-1.2 \% .{ }^{(5)}$ Patients afflicted with the condition often experience initial pain and inflammation at the affected joint, which gradually progresses to a loss of ROM over an extended period of time. ${ }^{(1-5)}$ In the advanced stage of $\mathrm{HO}$, patients eventually end up with an ankylosed joint.

For the affected patient, it is crucial that this condition is recognised and diagnosed early so that ankylosis can be prevented. $\mathrm{HO}$, in both the early and advanced stages, is known to mimic the radiological findings of other diseases. ${ }^{(6,7)}$ Hence, it is important that clinicians understand the radiological features of the condition, in correlation to the patient's clinical findings. Herein, we report a rare case of neurogenic $\mathrm{HO}$ occurring in the non-paretic limb of a post-stroke patient, and discuss its diagnostic challenges with respect to radiological imaging.

\section{CASE REPORT}

A 57-year-old Chinese woman presented with a two-week history of a painful lump on her right hip. She had a history of a right hemispheric stroke in 2002, which left her bedbound with a disabling left hemiplegia ever since. Post stroke, she underwent a few years of physiotherapy but remained bedbound, requiring assistance in her daily activities. She did not have any prior trauma, past history of malignancy, or any systemic symptoms such as fever.

Clinical examination of the right hip revealed a rigid, bonyhard mass that was inseparable from the proximal femur. This mass, which measured approximately $5 \mathrm{~cm} \times 5 \mathrm{~cm}$, was neither warm nor tender. The right hip was ankylosed at $30^{\circ}$ of flexion, $10^{\circ}$ of internal rotation and $10^{\circ}$ of adduction. The patient also had disuse atrophy of the musculature of both the upper and lower limbs. Laboratory tests revealed a normal white cell count and inflammatory markers that were not elevated. The patient's serum calcium and alkaline phosphatase levels were within normal limits.

Plain radiography performed at admission showed extensive ossification around the right hip joint, with minimal periosteal reaction. The hip appeared ankylosed. The right femur appeared to be internally rotated, with intact cortical congruity and no evidence of fracture or bony destruction (Fig. 1). Similarly, review of a prior computed tomography (CT) image taken in 2010 showed no evidence of hip fracture, although minimal ectopic bone formation was noted (images not shown). Magnetic resonance $(M R)$ imaging of the right hip showed the lesion with signal intensity similar to that of marrow fat. The lesion followed a course from the iliacus to the lesser trochanter, which was compatible with the clinical findings of bony ankylosis. The hypointense rim corresponded to the peripheral ossification seen in the plain radiographs (Figs. 2a \& b), and the findings were compatible with $\mathrm{HO}$. No invasion of the surrounding structures was observed.

${ }^{1}$ Department of Orthopaedic Surgery, ${ }^{2}$ Department of Diagnostic Radiology, Tan Tock Seng Hospital, Singapore

Correspondence: Dr Chong Han Pek, Medical Officer, Department of Orthopaedic Surgery, Tan Tock Seng Hospital, 11 Jalan Tan Tock Seng Singapore 308433. chonghan.pek@mohh.com.sg 


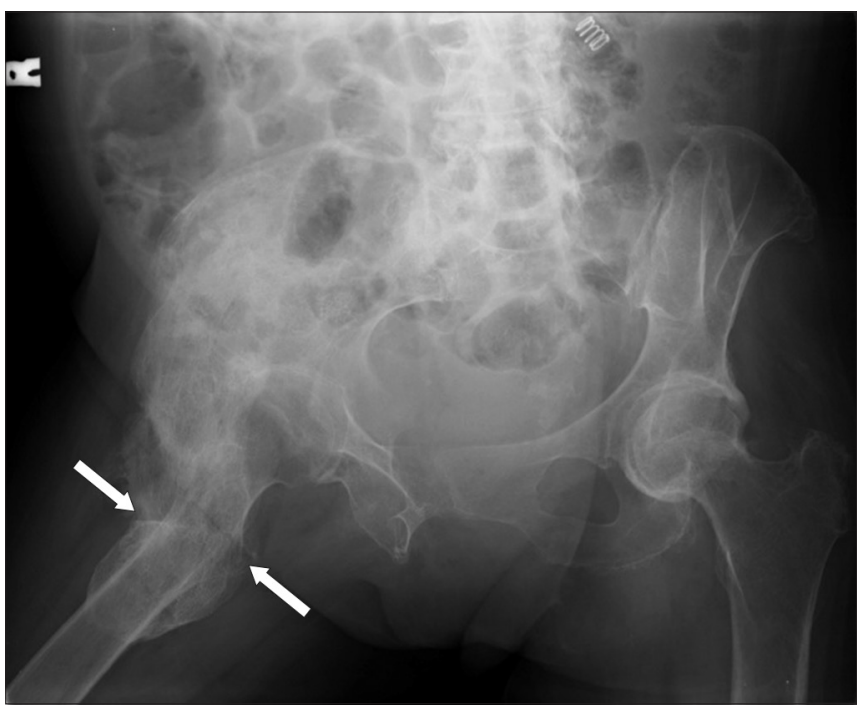

Fig. 1 Frontal radiograph of the pelvis shows florid ectopic bone formation around the right hip joint, causing bony ankylosis (arrows). The right femur appeared internally rotated, with preserved cortical congruity and no evidence of fracture or bony destruction demonstrated.
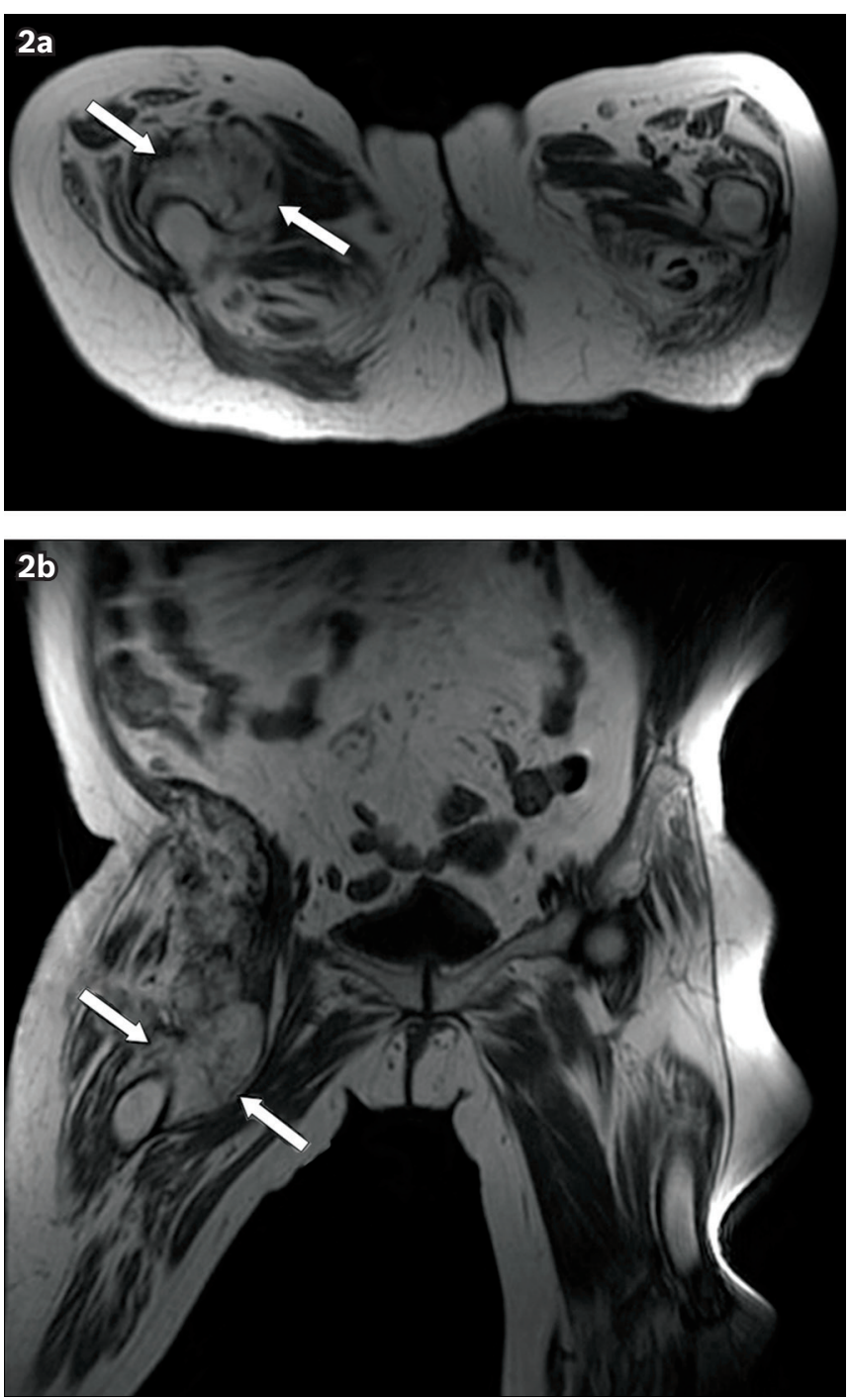

Fig. 2 (a) Axial and (b) coronal T1-W MR images of the pelvis show a fairly well-defined and lobulated mass in the anterior compartment of the right thigh abutting the adjacent muscles (arrows). The lesion demonstrates predominantly high T1-W signal hyperintensity, isointense to marrow fat, and hypointense signal is noted at the margins of the lesion.
Table I. Classification of $\mathrm{HO}$ according to radiological findings. ${ }^{(4)}$

\begin{tabular}{ll}
\hline HO classification & Radiological findings \\
\hline Class I & $\begin{array}{l}\text { Islands of bone within the soft tissue } \\
\text { around the hip } \\
\text { Bone spurs from the pelvis or proximal } \\
\text { Class II }\end{array}$ \\
& $\begin{array}{l}\text { opposing bone surfaces } \\
\text { Bone spurs from the pelvis or proximal end } \\
\text { Of the femur, reducing the space between } \\
\text { opposing bone surfaces to }<1 \mathrm{~cm}\end{array}$ \\
& Apparent bony ankylosis of the hip \\
Class IV &
\end{tabular}

The patient's family members, upon further questioning, conceded that they did not notice the presence of the right hip lump until the patient complained about her symptoms, and hence, they could not rule out the possibility that the lump was already there prior to the patient's complaints. They also maintained that the patient did not suffer any prior fall or trauma, as she had been bedbound since her stroke in 2002. Subsequently, the patient was placed in a community hospital where she received long-term physiotherapy. She was prescribed oral analgesics for pain relief, and her pain resolved after a few days. Surgical management was not offered for her ankylosed right hip, as it would not have improved her quality of life.

\section{DISCUSSION}

$\mathrm{HO}$ has been described to be mainly post-traumatic and neurogenic in origin. ${ }^{(3,8)}$ It has been suggested that posttraumatic HO commonly occurs after total hip arthroplasty. Brooker et al classified post-traumatic $\mathrm{HO}$ according to its radiological findings (Table I). ${ }^{(4)}$ Mavrogenis et al further classified neurogenic $\mathrm{HO}$ based on the anatomical location of $\mathrm{HO}$ : Type I - anterior; Type II - posterior; Type III - anteromedial; and Type IV - circumferential. ${ }^{(8)}$ Subtypes of each classification were then added based on spinal cord injury and brain injury. ${ }^{(8)}$ Knowledge of the different classifications of $\mathrm{HO}$ is important when considering surgical intervention, in relation to operative approach and preoperative planning.

Clinically, patients with $\mathrm{HO}$ may present with a spectrum of symptoms, ranging from pain with limitation of ROM to ankylosis of the affected joint. Such symptoms may not directly correlate with radiological findings. As such, clinical diagnosis of $\mathrm{HO}$ can be challenging. HO may also mimic different diseases at its various stages. Diagnosing $\mathrm{HO}$ using currently available imaging techniques is fraught with multiple challenges. We highlight some of these potential pitfalls in the interpretation of various imaging modalities.

Conventional radiography is commonly utilised at initial evaluation of patients with clinical symptoms suggestive of $\mathrm{HO}$. Typically, a soft tissue mass or swelling is the earliest radiographic finding. Ectopic bone formation can be seen as early as two weeks following surgery. Classically, $\mathrm{HO}$ is described as a peripheral zone of calcification with a relatively lucent centre, typically by 6-8 weeks post-surgery. The lesion tends to appear smaller and denser in the subsequent four months. ${ }^{(8)}$ 
On CT, lesion appearances usually parallel those on conventional radiographic images, i.e. bone formation that begins peripherally and ends centrally. As the lesion undergoes further maturation, a zonal pattern of mineralisation with the presence of a mature bony cortex is characteristically seen. There is high specificity for $\mathrm{HO}$ when such classic zonal appearance is visualised. ${ }^{(9,10)}$ Cross-sectional imaging is useful in the localisation of the lesion. However, if CT is done during the earlier stages of maturation, it may show a soft tissue mass with patchy or absent mineralisation, which may mimic a soft tissue sarcoma or osteosarcoma (particularly the juxtacortical subtype). ${ }^{(7)}$ Repeat CT after several weeks is useful, as it allows for better characterisation upon further maturation and evolution of the lesion.

MR imaging results vary according to the age of the lesion. Early features are nonspecific - heterogeneous high T2-weighted signal is frequently seen within the lesion and a hypointense rim representing calcification may be seen, although this is often indistinct. Intravenous gadolinium administration results in early, intense, heterogeneous enhancement of the lesion, which may be mistaken for osteomyelitis. ${ }^{(7,11,12)}$ After the lesion has undergone several weeks to months of progressive maturation, MR imaging may demonstrate a better-defined hypointense rim corresponding to the mature cortical bone, as well as high T1- and T2-weighted signals developing centrally (Fig. 3). ${ }^{(12)}$ Late lesions typically do not enhance, but some may enhance minimally (Fig. 4). The appearance of the lesions on MR imaging may mimic radiological findings of atypical lipomatous lesions such as liposarcomas. ${ }^{(11,12)}$ Triphasic radionuclide bone imaging is not recommended for the diagnosis of $\mathrm{HO}$. Although bone imaging is highly sensitive in the early stage of $\mathrm{HO}$, the technique has low specificity, as any local or systemic process that increases bone turnover can result in increased uptake. ${ }^{(13)}$

The cause of $\mathrm{HO}$ in our patient is likely to be microtrauma as a result of repeated cycles of prolonged immobilisation, followed by forcible mobilisation of the joints. ${ }^{(5,14)}$ Interestingly, HO occurred in the patient's non-paretic limb and the contralateral hip afflicted by the stroke is not affected. Such florid changes in the patient's non-paretic limb were unexpected. A possible hypothesis for such a phenomenon is that physiotherapy was more focused on the paretic limb, and thus, the non-paretic limb may have been unintentionally neglected. We were, however, unable to verify the hypothesis with the patient or her family members.

Management of $\mathrm{HO}$ is often targeted at preventing the condition from occurring in unaffected patients. Single-dose radiotherapy or oral nonsteroidal anti-inflammatory drugs such as indometacin have been described to be helpful in the prevention of $\mathrm{HO} .{ }^{(3,14,15)}$ Mavrogenis et al advocate resting the affected joint during the acute inflammatory stage of $\mathrm{HO}$, followed by passive ROM exercises and continued mobilisation once the inflammation subsides. ${ }^{(14)}$ Medical therapy and radiotherapy are available for the treatment and prophylaxis of $\mathrm{HO}$, but excisional surgery remains the treatment of choice to restore joint mobility. ${ }^{(1,3,14,15)}$ Due to the extensive mature $\mathrm{HO}$, our patient was managed with only oral analgesics. Surgical management was not indicated for her, as it would not have improved her mobility due to her prior stroke.

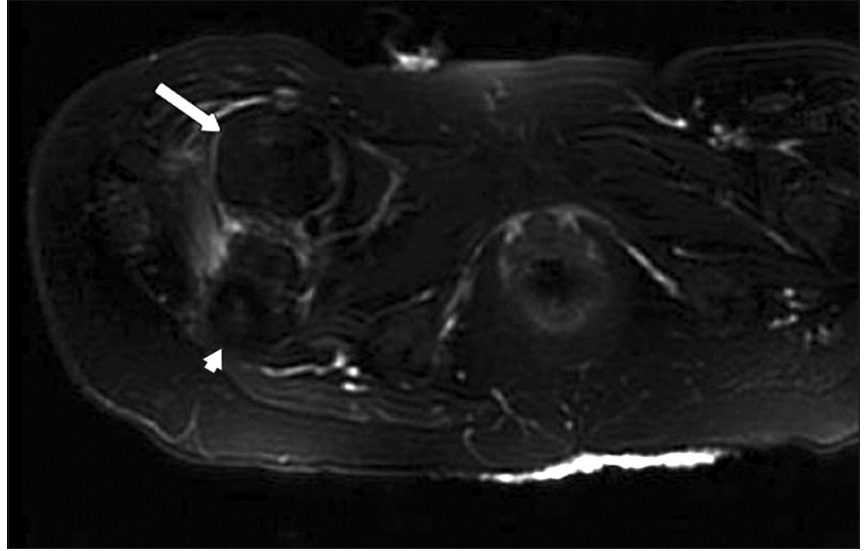

Fig. 3 Axial T2-W MR image with fat-suppression shows lesion signal that is completely suppressed (arrow) and signal intensity similar to the adjacent marrow (arrowhead).

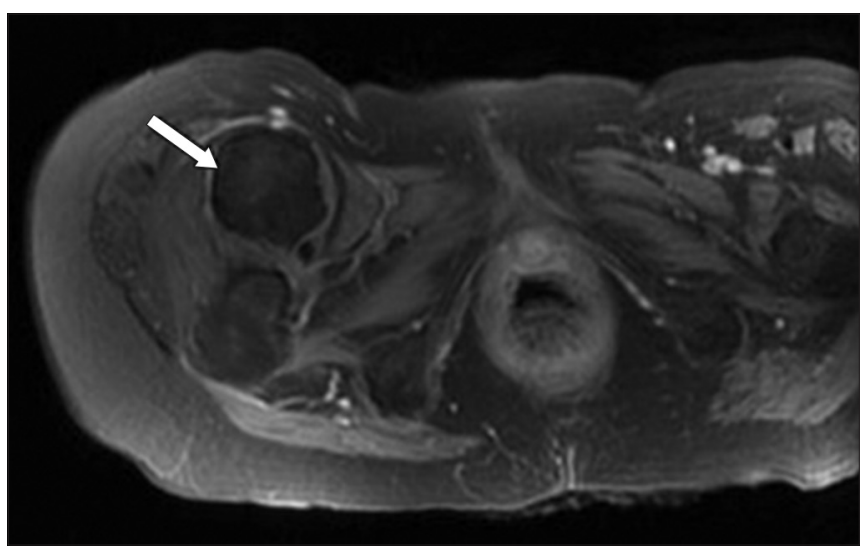

Fig. 4 Axial post-contrast T1-W MR image with fat-suppression shows no enhancement of the lesion (arrow).

In conclusion, neurogenic $\mathrm{HO}$ is commonly seen after a spinal cord or traumatic brain injury, but less commonly after a stroke. Due to the nonspecific nature of the radiological findings of early $\mathrm{HO}$, this clinical condition is easily overlooked and may hence be underdiagnosed. The present case is rare, as the patient's HO occurred in her non-paretic limb. Our patient had bony ankylosis of her right hip joint with radiological findings of extensive mature ossification of the periarticular soft tissue. The corresponding MR images, along with plain radiographs, showed extensive mature $\mathrm{HO}$, as the marrow within the ossification correlated with high T1-weighted signal hyperintensity seen on MR imaging. Thus, the diagnosis of $\mathrm{HO}$ could be made with certainty without any histological correlation. In terms of imaging, the technique of choice for our patient was $\mathrm{CT}$, as it enables better assessment of the osseous nature of the lesion. MR imaging, whilst superior in soft tissue definition, is less useful in the evaluation of $\mathrm{HO}$, as signal changes that are similar to marrow fat may mimic lipomatous tumours, particularly if the low signalintensity rim representing the bone is not evident in the image. ${ }^{(7,15)}$

\section{REFERENCES}

1. Genêt F, Jourdan C, Schnitzler A, et al. Troublesome heterotopic ossification after central nervous system damage: a survey of 570 surgeries. PLoS One 2011; 6:e16632.

2. Devnani AS. Management of heterotopic ossification affecting both hips and knees. Singapore Med J 2008; 49:501-4. 
3. Garland DE. A clinical perspective on common forms of acquired heterotopic ossification. Clin Orthop Relat Res 1991; 263:13-29.

4. Brooker AF, Bowerman JW, Robinson RA, Riley LH Jr. Ectopic ossification following total hip replacement. Incidence and a method of classification. J Bone Joint Surg Am 1973; 55:1629-32.

5. Crawford CM, Varghese G, Mani MM, Neff JR. Heterotopic ossification: are range of motion exercises contraindicated? J Burn Care Rehabil 1986; 7:323-7.

6. Kocaağa Z, Bal S, Gurgan A. Hemiplegia and heterotopic ossification on the non-paretic extremity: a case report. J Rehabil Med 2007; 39:500-2.

7. Kransdorf MJ, Meis JM. From the archives of the AFIP. Extraskeletal osseous and cartilaginous tumors of the extremities. Radiographics 1993; 13:853-84.

8. Mavrogenis AF, Guerra G, Staals EL, Bianchi G, Ruggieri P. A classification method for neurogenic heterotopic ossification of the hip. J Orthop Traumatol 2012; 13:69-78.

9. Bressler EL, Marn CS, Gore RM, Hendrix RW. Evaluation of ectopic bone by CT. AJR Am J Roentgenol 1987; 148:931-5.

10. Amendola MA, Glazer GM, Agha FP, et al. Myositis ossificans circumscripta: computed tomographic diagnosis. Radiology 1983; 149:775-9.

11. Ledermann HP, Schweitzer ME, Morrison WB. Pelvic heterotopic ossification: MR imaging characteristics. Radiology 2002; 222:189-95.

12 Kransdorf MJ, Meis JM, Jelinek JS. Myositis ossificans: MR appearance with radiologic-pathologic correlation. AJR Am J Roentgenol 1991; 157:1243-8.

13. Shehab D, Elgazzar AH, Collier BD. Heterotopic ossification. J Nucl Med 2002; 43:346-53.

14. Mavrogenis AF, Soucacos PN, Papagelopoulos PJ. Heterotopic ossification revisited. Orthopedics 2011; 34:177.

15. Javery O, Jagannathan JP, Saboo SS, et al. Case report: atypical lipomatous tumor with unusual extensive metaplastic ossification. Cancer Imaging 2012; 12:25-30. 